ORIGINAL ARTICLE

\title{
Intestinal Obstruction in Early Neonatal Period: A 3-Year Review Of Admitted Cases from a Tertiary Hospital in Ethiopia
}

\author{
Mustefa Mohammed ${ }^{1}$, Tadesse Amezene ${ }^{2^{*}}$, Moges Tamirat ${ }^{3}$
}

\section{OPEN ACCESS}

Citation: Mustefa Mohammed, Tadesse Amezene, Moges Tamirat. Intestinal Obstruction in Early Neonatal Period: A 3-Year Review Of Admitted Cases from a Tertiary Hospital in Ethiopia. J Health Sci 2017;27(4):393. http://dx.doi.org/10.4314/ejhs.v27i4.10

Received: February 27, 2017

Accepted: February 28, 2017

Published: July 1, 2017

Copyright: @ 2017 Mustefa Mohammed, et al. This is an open access article distributed under the terms of the Creative Commons Attribution License, which permits unrestricted use, distribution, and reproduction in any medium, provided the original author and source are credited.

Funding: Nil

Competing Interests: The authors

declare that this manuscript was approved by all authors in its form and that no

competing interest exists.

Affiliation and Correspondence:

${ }^{1}$ Ayder Hospital, Pediatrics and Child

Health Department, College of Health

Sciences, Mekele University, Ethiopia

${ }^{2}$ Department of Surgery, School of

Medicine, Addis Ababa University, Ethiopia

${ }^{3}$ Depatment of Pediatrics and Child

Health, Addis Ababa University, Ethiopia

"Email: amezenet@yahoo.com,

amezene.tadesse@gmail.com

\section{ABSTRACT}

BACKGROUND: Failure to pass meconium by a full-term neonate within the first 24 hours should raise a suspicion of bowel obstruction. The objective of this study was to determine pattern of presentation, diagnosis and outcome of management of intestinal obstruction in the early neonatal period in the Neonatal Care Unit of Tikur Anbessa Specialized Hospital in Addis Ababa, Ethiopia METHODS: Retrospective chart review of admitted cases from January 2011 to December 2013 was done. Data were entered into SPSS software version 20. Descriptive analysis of data were done at first; then cross tabulation of variables with chi-square tests were done

RESULT: Fifty-one (41 males and 10 females) cases of neonatal intestinal obstructions were described. ARM was the most common type of intestinal obstruction followed by intestinal atresia and Hirschsprung's disease.

Delayed diagnosis of cases was observed in $72 \%$. Associated congenital anomalies were documented in $13 \%$ of the cases. Death occurred in $20 \%$ of the cases. One or another form of infection accounted for $90 \%$ of the deaths. Neonatal sepsis and being unfit for anesthesia were determinants of mortality with p-value $<0.02$ and $<0.0007$ respectively.

CONCLUSION: Anorectal malformation is the commonest cause of early neonatal obstruction followed by small intestinal atresia and Hirschsprung's disease. Low number of associated anomalies has been reported. High rate of death due to preventable causes such as sepsis is observed. Delayed diagnosis of cases were also prevalent.

KEYWORDS: Neonatal intestinal obstruction, Anorectal malformation, Hirschsprung's Disease

\section{INTRODUCTION}

Failure to pass meconium by a full-term neonate within the first 24 hours should raise a suspicion of bowel obstruction. The commonest reported causes of bowel obstruction in decreasing order includes 
anorectal malformations, duodenal atresia, jejunoilealatresia, Hirschsprung's disease, meconium ileus and meconium plug syndrome(1). Other neonatal medical conditions like hypothyroidism, hypokalemia, sepsis and congestive heart failure are also reported to cause bowel obstruction (2).There are limited reports on the profiles of neonatal intestinal obstruction inEthiopia $(3,4)$. However, a number of studies have been cited to show the pattern of neonatal intestinal obstruction and determinants of mortality in other parts of the world $(5,6)$.

Although reports in developing countries are few, available evidence showed that neonates in African countries often do not get definitive medical care for several days. For example, one study from Nigeria emphasized problems of late presentation and poor neonatal ICU care facilities $(5,7)$. The purpose of this study is to review the pattern of intestinal obstruction in the early postnatal period, its associated conditions and outcome of management in order to determine factors associated with death of these newborns in our hospital.

\section{MATERIALS AND METHODS}

Study site: The study was conducted at the neonatal intensive care unit (NICU) of Tikur Anbessa Specialized Hospital (TASH) which is the main neonatal care center in Ethiopia. TASH is the tertiary university hospital located in the capital city, Addis Ababa, where all neonatal intestinal obstruction cases are referred to. Cases under the age of eight days were admitted to the unit, as it is the unit's protocol since its establishment. Admission of cases is done after consulting the Pediatric Surgery Department. The unit is equipped with incubators and CPAP machines. The unit does not have respiratory support with mechanical ventilation and total parenteral nutrition (TPN) which are important for survival of neonates with intestinal obstruction.

Study design and period: A retrospective chart review of cases admitted in the period of January 2011 to December 2013 was performed.

Study population: Cases of intestinal obstruction in the first 7 days of birth admitted in the specified time period were included. Cases with incomplete records were excluded from the study.

Study variables: Age and sex of patients, age at presentation, presenting symptoms, time of diagnosis, birth weight, gestational age, presence of associated congenital anomaly, associated Down's syndrome, type of intestinal obstruction, type of surgical intervention, pre- and postoperative complication, factors determining mortality and discharge outcome were the variables of this study.

Data collection: Data were collected from patients' charts on to structured questionnaire form.

Statistical analysis: Data were entered into SPSS software version 20. (IBM 2012). Descriptive analysis of data were done at first; then cross tabulation of variables with chi-square tests was done. Fisher's exact value were used whenever value < five occurred in the data. P-value $<0.05$ was used to indicate significant value.

Ethical consideration: The study obtained ethical clearance from Research and Publication Committee of the Department of Pediatrics and Child Health at School of Medicine, Addis Ababa University. In order to secure confidentiality unique identifiers like names and telephone numbers were not recorded. Data were kept in the computer using password protection by the principal investigator.

\section{RESULTS}

Fifty-one cases were described. All cases were delivered outside of TASH except one. A total of 8805 neonatal (0-7 days) admissions were recorded in the specified time period, out of which intestinal obstruction accounted for $0.6 \%$. The mean age at admission was 73 hours. The mean durations of symptoms were 2.5 days, 3.5 days and 5 days in the case of intestinal atresia, anorectal malformation and Hirschsprung's disease, respectively. The sex distribution showed male predominance with male to female ratio of 4.6:1. There was one case in which the gender identity was not determined clinically (ambiguous genitalia).

DOI: http://dx.doi.org/10.4314/ejhs.v27i4.10 
Anorectal malformation was the most common type of neonatal intestinal obstruction, 29(57\%) of which $16(55 \%)$ low type and $13(45 \%)$ high type, respectively. The other causes include intestinal atresia, 7(14\%), Hirschsprung's disease, 6(12\%), and others, 9(17\%). Unspecified diagnosis includes cases of intestinal malrotation in $3(5.9 \%)$, meconium plug in $2(3.9 \%)$, congenital adhesion band in $1(2 \%)$, colonic atresia in $1(2 \%)$, paralytic ileus secondary to hypokalemia in $1(2 \%)$, and spontaneous gastric perforation in $1(2 \%)$ patient.

Delayed presentation and associated congenital anomalies occurred in 37 cases (72\%) and in seven cases (13.7\%), respectively. Failure to pass meconium,

Table 1: Patient characteristics of intestinal obstruction in the early neonatal period TASH 2011-2013.

\begin{tabular}{|c|c|c|}
\hline Variable & Categories & Percentage \\
\hline All & & $51(100)$ \\
\hline \multirow[t]{2}{*}{ Sex } & Male & $41(80)$ \\
\hline & Female & $10(20)$ \\
\hline \multirow[t]{4}{*}{ Age at presentation } & Day 1 & $7(14)$ \\
\hline & Day 2-3 & $24(47)$ \\
\hline & Day 4-7 & $20(39)$ \\
\hline & After day 7 & $0(0)$ \\
\hline \multirow[t]{7}{*}{ Diagnosis } & Anorectal Malformation & $29(57)$ \\
\hline & Atresia & $7(13)$ \\
\hline & Hirschsprung's disease & $6(12)$ \\
\hline & Malrotation & $3(6)$ \\
\hline & Hypokalemia/meconium ileus & $2(4)$ \\
\hline & Gastric perforation & $1(2)$ \\
\hline & Other & $3(6)$ \\
\hline \multirow[t]{6}{*}{ Associated conditions } & VACTREL association & $4(31)$ \\
\hline & Tracheo-esophageal fistula & $2(15)$ \\
\hline & Congenital Heart disease & $2(15)$ \\
\hline & Ambiguous genitalia & $1(7.5)$ \\
\hline & Hypospadias & $1(7.5)$ \\
\hline & Non-visualized right kidney & $1(7.5)$ \\
\hline \multirow[t]{4}{*}{ Surgical intervention } & Colostomy & $34(67)$ \\
\hline & Definite surgery & $13(25)$ \\
\hline & No operation & $4(8)$ \\
\hline & Missing information & $0(0)$ \\
\hline \multirow[t]{4}{*}{ Final outcome } & Death & $10(20)$ \\
\hline & Discharge cured & $41(80)$ \\
\hline & Discharged & $34(66.6)$ \\
\hline & withdisability(colostomy) & \\
\hline \multirow[t]{6}{*}{ Immediate cause of death } & Sepsis & $9(90)$ \\
\hline & Apnea with sepsis & $1(10)$ \\
\hline & DIC with sepsis & $1(10)$ \\
\hline & CHF without sepsis & $1(10)$ \\
\hline & Pneumonia with sepsis & $1(10)$ \\
\hline & Aspiration with sepsis & $1(10)$ \\
\hline
\end{tabular}

DOI: http://dx.doi.org/10.4314/ejhs.v27i4.10 
abdominal distension and vomiting each shared a third of the presenting symptoms. Low birth weight (all forms) and preterm deliveries were seen in 12 and 7 of the cases respectively. Out of the 6 cases of jejunoileal atresia, two were preterm. Four of the 6 cases were low birth weight, with one of them being small for gestational age. There was one duodenal atresia (2\%) with Down syndrome and low birth weight. Two cases had small intestinal obstruction secondary to adhesion band.

Surgery was performed on 47 cases (92.8\%). Colostomy, anoplasty, resection with end-to-end anastomosis were done in 34 cases (66.7\%), $1(2 \%)$ and $12(23.5 \%)$ of the cases, respectively. Four neonates $(7.8 \%)$ were not operated on because three of them were critically sick and the fourth had meconium plug syndrome, which was managed with rectal saline washout. Postoperative complications included wound infection in $4(8.7 \%)$, aspiration in $1(2 \%)$, apnea in $1(2 \%)$. One patient $(2 \%)$ required re-operation. Ten $(19.6 \%)$ cases died in the hospital.

Associated congenital anomalies include VACTREL association, tracheo-esophageal fistula, congenital heart disease, ambiguous genitalia, hypospadias and non-visualized right kidney (Table 1). Delay in presentation and sepsis are factors that determine mortality (Table 2).The clinical characteristics of mortality cases are shown in Table 3. Neither preoperative abdominal ultrasound nor blood culture were done in all of the cases.

Table 2: Determinants of mortality, intestinal obstruction in the early neonatal period. TASH 2011- 2013

\begin{tabular}{|c|c|c|c|c|}
\hline Variable & Categories & Number & Percentage & P-value \\
\hline \multirow[t]{2}{*}{ Sex } & Male & 44 & $9(21)$ & 0.5 \\
\hline & Female & 7 & $1(14)$ & \\
\hline \multirow[t]{3}{*}{ Delayed diagnosis } & Yes & 37 & $5(14)$ & 0.09 \\
\hline & No & 9 & $5(56)$ & \\
\hline & Missing & & & \\
\hline \multirow[t]{3}{*}{ Gestation } & Term & 44 & $9(21)$ & 0.5 \\
\hline & Preterm & 7 & $1(14)$ & \\
\hline & Missing & & & \\
\hline \multirow[t]{3}{*}{ Associated conditions } & Yes & 7 & $3(45)$ & 0.1 \\
\hline & No & 44 & $7(16)$ & \\
\hline & Missing & & & \\
\hline \multirow[t]{3}{*}{ Sepsis } & Yes & 21 & $9(43)$ & 0.0007 \\
\hline & No & 30 & $1(3)$ & \\
\hline & Missing & & & \\
\hline \multirow[t]{3}{*}{ Body weight } & Not SGA & 46 & $8(17)$ & 0.25 \\
\hline & SGA & 5 & $2(40)$ & \\
\hline & Missing & & & \\
\hline \multirow[t]{3}{*}{ Intervention } & Yes & 47 & $7(15)$ & 0.02 \\
\hline & No & 4 & $3(75)$ & \\
\hline & Missing & & & \\
\hline \multirow{5}{*}{ Obstruction type } & Anorectal malformation & 29 & $8(28)$ & 0.1 \\
\hline & Atresia & 7 & $2(29)$ & \\
\hline & Hirschsprung's disease & 6 & 0 & \\
\hline & Malrotation & 3 & 0 & \\
\hline & Others & 6 & 0 & \\
\hline
\end{tabular}

DOI: http://dx.doi.org/10.4314/ejhs.v27i4.10 
Table 3: Profiles of mortality cases, intestinal obstruction in early neonatal period. TASH.2011-2013

\begin{tabular}{|c|c|c|c|c|c|}
\hline Sex & $\begin{array}{l}\text { Age at } \\
\text { presentation }\end{array}$ & Diagnosis & $\begin{array}{l}\text { Associated } \\
\text { conditions }\end{array}$ & Management & $\begin{array}{l}\text { Immediate } \\
\text { cause of death }\end{array}$ \\
\hline $\mathrm{M}$ & 3 days & Low ARM & VACTERL & colostomy & Sepsis, apnea \\
\hline M & 2 days & High ARM & VSD & Colostomy & Sepsis, DIC \\
\hline M & 1 day & Illeal atresia & No & Definitive surgery & Sepsis \\
\hline M & 1 day & Jejunal atresia & No & Definitive surgery & Sepsis \\
\hline M & 1 day & $\begin{array}{l}\text { LowARM } \\
\text { Perineal fistula- } \\
\text { Bifid scrotum- } \\
\text { SGA }\end{array}$ & $\begin{array}{l}\text { VACTERL } \\
\text { ASD } \\
\text { PHT }\end{array}$ & Not operated & $\begin{array}{l}\text { Congestive heart } \\
\text { failure }\end{array}$ \\
\hline M & 2 days & $\begin{array}{l}\text { High ARM } \\
\text { Malrotation -band }\end{array}$ & No & $\begin{array}{l}\text { colostomy- } \\
\text { laparotomy }\end{array}$ & Sepsis \\
\hline M & 1 day & $\begin{array}{c}\text { LowARM } \\
\text {-VVF }\end{array}$ & $\begin{array}{l}\text { TEF } \\
\text { VACTERL }\end{array}$ & Not operated & $\begin{array}{l}\text { Aspiration } \\
\text { Pneumonia }\end{array}$ \\
\hline M & 1 day & $\begin{array}{l}\text { High ARM } \\
\text { Anemia }\end{array}$ & No & Not operated & Sepsis \\
\hline M & 4 days & $\begin{array}{l}\text { High ARM- } \\
\text { Anemia, } \\
\text { Hypocalcemia }\end{array}$ & VACTERL & Colostomy & Sepsis \\
\hline M & 3 days & High ARM & No & Colostomy & Sepsis \\
\hline
\end{tabular}

\section{DISCUSSION}

We described 51 neonates in the first week of life who suffered from various types of intestinal obstructions and their associated congenital malformations. Anorectal malformation is the most common diagnosis made. Colostomy is the most common procedure done in the unit and high rate of death recorded, mainly related to infection. Although there were separate reports of anorectal malformation (11) and neonatal gastrointestinal malformations $(3,4)$, this is the first review of early neonatal intestinal obstruction from Ethiopia. Male sex is reported more commonly than female sex, a finding consistently observed in many other reports too $(5,8,9)$. However, the difference in sex in the present study is much higher than the others. It has been claimed that imperforate anus may be missed in female neonates in whom meconium is passed through a fistula communicating the rectum with the perineum and/or vestibule. Such a possibility of missing ARM in female sex has been the concern of others, and it has been recommended to do focused physical examination of the anal area in all suspected cases (7). EA Ameh reported significantly large proportion of high type ARM compared to ours, which is more dominated by Low type ARM (5).

Similar report with ours were made by Masao Endo from Japan with regard to type of ARM (9). On the other hand, a striking difference occurred between the Nigerian study and ours. They had $86.5 \%$ of their ARM cases being high type, where us only $44.8 \%$ of our cases were high type. These have implications both in the type and outcome of of surgery. Proper evaluation of cases both before and during surgery is essential $(5,10)$.

According to literatures, neonates who do not pass meconium for more than 48 hours after birth are at moderate risk for having Hirschsprung's disease (approximately 5-20 percent) and should be evaluated by a health care professional for this disorder (11). The diagnosis of Hirschsprung's disease is being increasingly made in the neonatal 
period in the developed countries, while the the reverse situation is still prevalent in developing countries as it is seen in Zaria and in Ethiopia as seen in this $\operatorname{study}(5,12)$.

In developed countries, small intestinal atresia is a common cause of congenital intestinal obstruction, and it accounted for one-third of causes of neonatal intestinal obstructions according to one report (7).We reported small intestinal atresia in $14 \%$ of the cases. The cases in our situation may die before they reach hospital for lack of early detection and referral. A similar argument has been given by others (13). Adequate training should be given to health extension workers as they are closer to the community.

The low prevalence of associated congenital anomalies in this study could be because of some missed cases. Tareen reported $80-100 \%$ associated congenital anomalies in their review (6), whereas C.stoll et al reported $49 \%$ of their cases to have associated anomalies. In the latter studies, urogenital system $(81.1 \%)$ and of the skeletal system $(45.5 \%)$ were the most common organs involved. VACTERL association is the commonest associated condition observed in our study $(6,14)$.

The consequence of delayed diagnosis was discussed by $\mathrm{F}$ Tareen et al (6). They reported 2 cases of bowel perforation as a result of delayed diagnosis. Another report by Haider $\mathrm{N}$ et al described rate of bowel perforation double that of F Tareen report $(6,15)$. Lindly believed that this problem of bowel perforation is more common in developing nations (16). One case of bowel perforation is reported in the current review.

High prevalence of clinical sepsis was found in those neonates who died. A similar observation was made by Ameh et al. They reported $70 \%$ of cause of death being infection. They also reported $21 \%$ mortality (5). Even though we do not have studies in the past, the present report of $20 \%$ hospital mortality is still high. However, we expect higher mortality if we were in a position to study cases up to the age of 28 days like they did. According to reports by Reyes HM, the overall mortality of neonates suffering from intestinal obstruction should be fewer than 5\%. High survival rate in their report is accounted for by improved care patients received in specialized units (17).

Nine of the 10 deaths reported in this study occurred due to one or another form of infection. The presence of unfitness to anesthesia seems to be a determinant factor for death in this study. A similar observation was also made by Millar HRG (18).

Associated congenital anomalies are low in our study. Various authors suggested that a number of congenital anomalies may be missed on routine neonatal checkup. Memberg $\mathrm{A}$ et al reported substantial proportion (24\%) of congenital cardiac malformations detected after discharge from hospital after birth (19). By the same token, $F$ Tareen reported a case of previously undiagnosed ARM but also missed case of Tetralogy of fallot (6). C.Stole et al reported that half of their study cases of ARM had associated malformations and recommended routine screening for congenital malformations and genetic counseling to be considered in patients with $\operatorname{ARM}(14)$.

One-third of the cases of Jejuno-illeal atresia occurred in preterm infants in our study. This finding is in accordance with the finding of Alastir (18). The case with duodenal atresia in the present study occurred in patienst with Down syndrome. It is well documented that $30 \%$ of duodenal atresia cases will have Down syndrome. Since we reported only one case, we did not have the chance to see more common associated anomalies as reported by others (20).On the other hand, Rescorla FJ, Grosfeld JL reported severe associated anomalies in 32 of their 39 cases of duodenal atresia cases (21). In order to prevent misdiagnosis of cases, James A suggested, in addition to careful perianal inspection, a cross table lateral prone $\mathrm{X}$-ray to be done $(22,23)$.

This study is robust in that the data used may be representative of the national picture as patients come from all over the country since TASH is the only tertiary center for pediatric surgery in Ethiopia. However, because of the poor referral system, this may not be always true. One of the limitation of the study may be neonates above the age of 7days were not included as the NICU admits cases only up to the age of 7 days.

DOI: http://dx.doi.org/10.4314/ejhs.v27i4.10 
Therefore, the information obtained may not be complete. The diagnosis of sepsis is mainly based on clinical evidences; this may also lead to incorrect diagnosis. Reasons for delayed diagnosis were not also inquired and documented.

In general, this review demonstrated a similar trend with other most reported cases in terms of delayed diagnosis, but abnormally high mortality, high degree of infection and under-diagnosis of associated conditions in neonatal intestinal obstruction cases in TASH. In conclusion, anorectal malformation is the commonest cause of early neonatal obstruction followed by small intestinal atresia and Hirschsprung's disease. Low number of associated anomalies has been reported. High rate of death due to preventable causes such as sepsis was observed. Delayed diagnosis of cases were also prevalent.

Future studies should focus on getting data on possible sources of neonatal sepsis, type of organisms and preventive measures that are not followed in the NICU. Careful perianal examination of all newborns should be a routine practice before the newborn is discharged from the hospital. Proper workup of associated anomalies and proper documentation of the findings is necessary as these may have an impact on the management and its outcome.

The authors would like to recommend careful screening of neonates for ARM and associated conditions before discharge from hospital. Once cases are diagnosed and admitted, the practice of washing hand in the NICU should be high standard in order to reduce risk of infection.

\section{REFERENCES}

1. Yu.F.Isakov,Yuranga Weerakkody, J. J. et. al. Duodenal atresia. Pediatric surgery 1988; http://radiopaedia.org/articles/duodenalatresia.

2. Vera Loening-Bauke, KK. Failure to Pass Meconium: Diagnosing Neonatal Intestinal Obstruction. Am Fam Physician. 1999; 60 (7): 2043-2050.

3. Endale Tefera, Telahun Teka, Milliard Derbew. Neonatal gastrointestinal surgical emergencies: a 5- year review in a teaching hospital in Addis Ababa, Ethiopia. Ethiop Med J 2007; 45( 3):2516.

4. Ameh EA, C. L. Neonatal intestinal obstruction in Zaria, Nigeria. East Afr Med J. 2000; 77: 510-513.

5. F Tareen, D. C., OM Aworanti, J GillickOur. Delayed Diagnosis of Anorectal Malformation - A Persistent Problem. Ir Med J. 2013; 106(8): 238-240.

6. Chirdan LB, U. A., Pam SD. Intestinal atresia: Management problem in developing country. paediatr surg Int. 2004; 20: 834837.

7. Miliard Derbew and Marc A Levitt. Newborn Management of Anorectal Malformations. Surgery in Africa monthly review. 2009; 7:1-14.

8. Masao Endo, A. H., Michiomi Ishihara, Masahiko Maie, Akira Nagasaki,Toshiji Nishi, Morihiro Saeki. Analysis of 1,992 patients with anorectal malformations over the past two decades in Japan. Journal of Pediatric Surgery. 1999;.34( 3 ): 435-441.

9. Feilim Murphy, P. P., John M. Hutson and Alexander M. Holschneider. Incidence and frequency of different types and Classification of Anorectal malformation. Embryology, Diagnosis, Surgical Treatment, Follow-up. by Holschneider AM, Malformations, Ed. 2006. New York, Springer; OpenURL.

10. Children's Hospital, 2015. Hirshsprungs disease.

http://www.stlouischildrens.org/diseasesconditions/hirschsprung-disease.

11. H. L. Nancy Kim KWG, J. G. P., Geoffrey K. Blair James J. Murphy, Eric M. Webber. Presentation of Low Anorectal Malformations Beyond the Neonatal Period. Pediatrics. 2000;105 (5): 68.

12. Philoppart A.l. Hirschsprung's disease. In: Ashcraft KW, H. T. e., Ed. Paediatric Surgery.1993,Philadelphia, WB Saunders Company.

13. Stoll C, Alembik Y, Dott B, Roth MP. Associated malformations in patients with

DOI: http://dx.doi.org/10.4314/ejhs.v27i4.10 
anorectal anomalies. Eur J Med Genet. 2007;50(4):281-90.

14. Haider N, FR. Mortality and morbidity associated with late diagnosis of anorectal malformations in children. Surgeon. 2007; 5: 327-330.

15. Lindley RM, S. R., Roberts JP. Delays in the diagnosis of anorectal malformations are common and significantlyincrease serious early complications. Acta Paediatr. 2006; 95:364-368.

16. Reyes HM1, M. J., Loeff D .Neonatal intestinal obstruction. Clin Perinatol.1989; 16(1): 85-96.

17. Alastair J.,W Millar HRG, K. L. Operative management of intestinal atresia and stenosis based on pathological findings. $J$ pediatr Surg. 1979; 14: 368.

18. Meberg A, Otterstad JE, Froland G, Hals J, Sorland SJ. Early clinical screening of neonates for congenital heart disease: the cases we miss. Cardiology Young. 1999; 9: 169-74.
19. Bales C. L., CA. Duodenal obstruction. In: Kliegman, RM, Behrman RE, St. Geme III JW, Schor NF, Stanton BF, 2013:1277-78.

20. Rescorla FJ, G. J. Intestinal atresia and stenosis: analysis of survival in 120 cases. Surgery. 1985; 98(4): 668-676.

21. Rosen NG, Hong AR, Soffer SZ, Rodriguez G, Peña A. Rectovaginal fistula: a common diagnostic error with significant consequences in girls with anorectal malformations. $J$ Pediatr Surg. 2002;37(7):961-5.

22. Rode H, M. A. Jejuno-ileal atresia and stenosis. In: PremPuri, ed. Newborn Surgery, 2nd ed. By Hodder Arnold.338 Euston Road, London NW1 3BH, 2003 pp 445-456.

23. James A. O'Neil, Jr. Anorectal disorders and imperforate anus. In Principles of Paediatric Surgery, 2004. pp 596-603. 